\title{
Preferential binding of lysozyme to elastic fibres in pulmonary emphysema
}

\author{
Bronislava Shteyngart, Surachat Chaiwiriyakul, John Wong, Jerome O Cantor
}

\begin{abstract}
Background-Lysozyme is increased in inflammatory reactions and is a component of the extracellular matrix, but its possible role in lung diseases such as emphysema and interstitial fibrosis has not been investigated.

Methods-To characterise differences in lysozyme content among normal, emphysematous, and fibrotic human lungs, tissue sections obtained from necropsy specimens were immunostained with rabbit polyclonal anti-human lysozyme antibody using the labelled streptavidin-biotin peroxidase method. The immunostained sections were evaluated semiquantitatively (grading the degree of immunostaining on a scale of 0-4). To determine if degradation of the extracellular matrix affects lysozyme binding, hyaluronidase-treated normal lung tissues were incubated with egg white lysozyme, immunostained with the lysozyme antibody, which crossreacts with egg white lysozyme, and evaluated for degree of staining.
\end{abstract}

Results-Lysozyme immunostaining was significantly increased in lungs with pulmonary emphysema compared with normal or fibrotic tissues (3.4 versus 1.6 and 1.9 , respectively; $p<0.05)$ and was preferentially associated with interstitial elastic fibres. Hyaluronidase-treated lung tissues incubated with lysozyme showed increased immunostaining for this protein compared with untreated controls (1.9 versus $1.2 ; \mathrm{p}<0.05)$.

Conclusions-The results suggest that damage to elastic fibres and/or the surrounding extracellular matrix increases lysozyme binding. It is hypothesised that attachment of lysozyme to elastic fibres may interfere with their repair and possibly enhance the progression of pulmonary emphysema.

(Thorax 1998;53:193-196)

Keywords: lysozyme; pulmonary emphysema; elastic fibres

This study examined the possible role of lysozyme in lung injury. The investigation was prompted by earlier studies indicating that lysozyme is increased in a number of inflammatory conditions and is specifically associated with extracellular matrix constituents. ${ }^{1-3}$ These findings suggested that the amount of lysozyme in the lung might be altered in diseases such as emphysema or interstitial fibrosis where there is damage to various matrix components.

To determine if lysozyme is involved in pulmonary disease, tissue sections from normal, fibrotic, and emphysematous human lungs were evaluated for differences in lysozyme content. An increase in extracellular lysozyme was specifically observed in lung tissues with pulmonary emphysema, and the protein was preferentially associated with elastic fibres which undergo breakdown in this disease. Since this laboratory and other investigators have previously shown that hyaluronic acid and other polysaccharides surround elastic fibres, ${ }^{45}$ normal lung tissues were treated with hyaluronidase and examined for their ability to bind exogenously administered lysozyme. Such treatment resulted in increased attachment of lysozyme, suggesting that degradation of extracellular matrix components, as occurs in pulmonary emphysema, may expose binding sites for lysozyme on elastic fibres, possibly altering matrix interactions and influencing the repair process in this disease.

\section{Methods}

PROCUREMENT OF NECROPSY LUNG TISSUES The necropsy files of The Brooklyn Hospital Pathology Department were reviewed to obtain tissues from normal $(\mathrm{n}=7)$, emphysematous $(n=7)$, and fibrotic lungs $(n=6)$. Tissues designated normal were obtained from patients with no lung complications at the time of death. Multiple slide sections (three or more) of the lungs were examined by a pathologist (JOC) to confirm the findings and immunohistochemical studies were then performed on sections of a representative paraffin-embedded tissue block from each case.

IMMUNOHISTOCHEMICAL STAINING FOR LYSOZYME

The labelled streptavidin-biotin peroxidase method was used to label lysozyme. Tissue sections were deparaffinised in xylene, hydrated through graded alcohols, then rinsed in 
$0.05 \mathrm{M}$ Tris- $\mathrm{HCl}$ buffer, $\mathrm{pH}$ 7.6. Following inhibition of endogenous peroxidase with $3 \%$ hydrogen peroxide, the tissue sections were washed with Tris- $\mathrm{HCl}$ buffer and incubated with $2 \%$ fetal bovine in Tris- $\mathrm{HCl}$ for 20 minutes to block non-specific protein binding. They were then incubated for 30 minutes with a polyclonal rabbit anti-human lysozyme antibody (BioGenex Laboratories, San Ramon, California, USA) and separately treated for 15 minutes with biotin-labelled link antibody and peroxidase-labelled streptavidin. Immunostaining was completed with a 20 minute incubation in 3-amino-9-ethylcarbazole (AEC) buffer solution. The tissues were then counterstained with Mayer's haematoxylin.

A positive control specimen of normal human tonsil accompanied each staining run to verify proper functioning of all reagents involved in the immunostaining procedure. Negative control specimens for each test case were also performed, substituting the antihuman lysozyme primary antibody with a nonimmune rabbit serum negative control reagent to determine the degree of non-specific staining.

EVALUATION OF IMMUNOSTAINING

The tissue sections were coded and independently graded for the degree of extracellular lysozyme immunostaining by a pathologist (SC) unfamiliar with the design of the experiment. The entire area of each tissue section was examined and only extracellular staining of the lung parenchyma was evaluated, since inflammatory cells (monocytes, macrophages, and neutrophils) showed intense staining which could obscure differences in lysozyme content related to interstitial connective tissue changes. Slide sections were given a score between 0 and 4. Intermediate scores were averaged (e.g. 1-2 = 1.5).

\section{HYALURONIDASE-LYSOZYME TREATMENT OF} LUNG TISSUES

Tissue sections ( $n=9$ ) from normal lungs were used in these studies (in some cases more than one paraffin block per lung was utilised). The sections were deparaffinised and coated with aliquots of a solution of bovine testicular hyaluronidase (Wyeth Laboratories, Philadelphia, Pennsylvania, USA) at a concentration of $150 \mathrm{U}$ per $\mathrm{ml}$ phosphate buffered saline. The tissues were treated for two hours at $37^{\circ} \mathrm{C}$. Control sections from the same tissue blocks were coated with phosphate buffered saline solution alone under similar conditions. Both groups were then incubated with a $1 \%$ solution of egg white lysozyme (Sigma Chemical Company, St Louis, Missouri, USA) in phosphate buffered saline for one hour at room temperature. After extensive rinsing, the tissues were immunostained for lysozyme.

The rabbit antibody, prepared with lysozyme isolated from the urine of patients with monocytic leukaemia, was tested for crossreactivity with egg white lysozyme. Samples of human neutrophil lysozyme and egg white lysozyme were spotted onto nitrocellulose paper, then

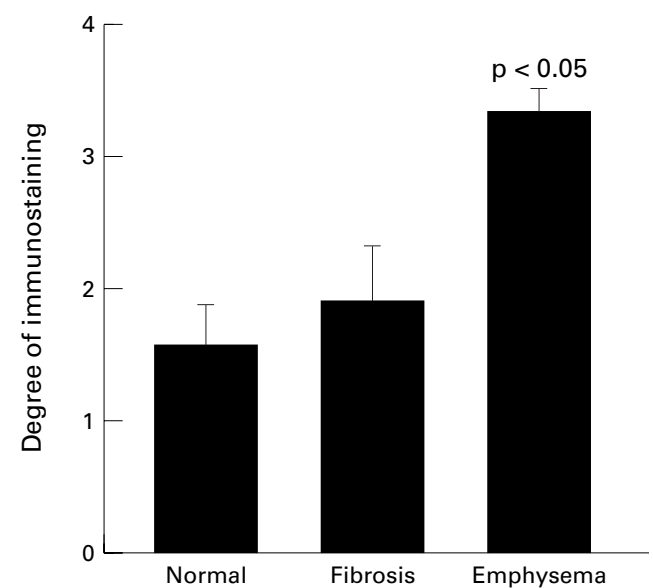

Figure 1 Mean (SE) immunostaining for lysozyme in pulmonary emphysema compared with both normal and fibrotic lungs.

incubated with the antibody and labelled as described above.

To determine the effect of hyaluronidase treatment alone on lysozyme immunostaining, tissue sections $(n=7)$ from normal lungs were incubated with the enzyme or with buffer alone, as described above. The tissues were then immunostained without prior incubation with egg white lysozyme.

\section{STATISTICAL ANALYSIS}

Differences in immunostaining among normal, emphysematous, and fibrotic lung tissues were analysed for statistical significance $(p<0.05)$ with the Kruskal-Wallis multiple comparison $z$ value test. The Wilcoxon signed rank test was used to evaluate differences between hyaluronidase-treated and untreated tissues.

\section{Results}

Emphysematous lungs showed a significant increase in extracellular immunostaining for lysozyme compared with both normal and fibrotic lung tissues (mean (SD) 3.4 (0.5) versus 1.6 (0.8) and 1.9 (1.0), respectively; $\mathrm{p}<0.05$; fig 1 ). In all three groups there was preferential staining of interstitial, vascular, and pleural elastic fibres. However, the emphysematous lungs showed particularly intense staining of these fibres, especially in areas where there was airspace dilatation and attenuation of the alveolar septa (fig 2). The immunostained elastic fibres associated with alveolar distention often appeared fragmented (fig 3).

Tissue sections from normal lungs treated with bovine testicular hyaluronidase and incubated with egg white lysozyme showed a significant increase in immunostaining for lysozyme compared with controls not exposed to hyaluronidase (1.9 (0.8) versus 1.2 (0.7), respectively; $\mathrm{p}<0.05)$. This result was not due to increased immunostaining of endogenous lysozyme, since tissue sections treated with hyaluronidase but not incubated with lysozyme showed no significant increase in immunostaining compared with controls $(0.9(0.2)$ versus $0.6(0.2)$, respectively; $p>0.05)$.

The anti-human lysozyme antibody used in these studies reacted positively when tested 

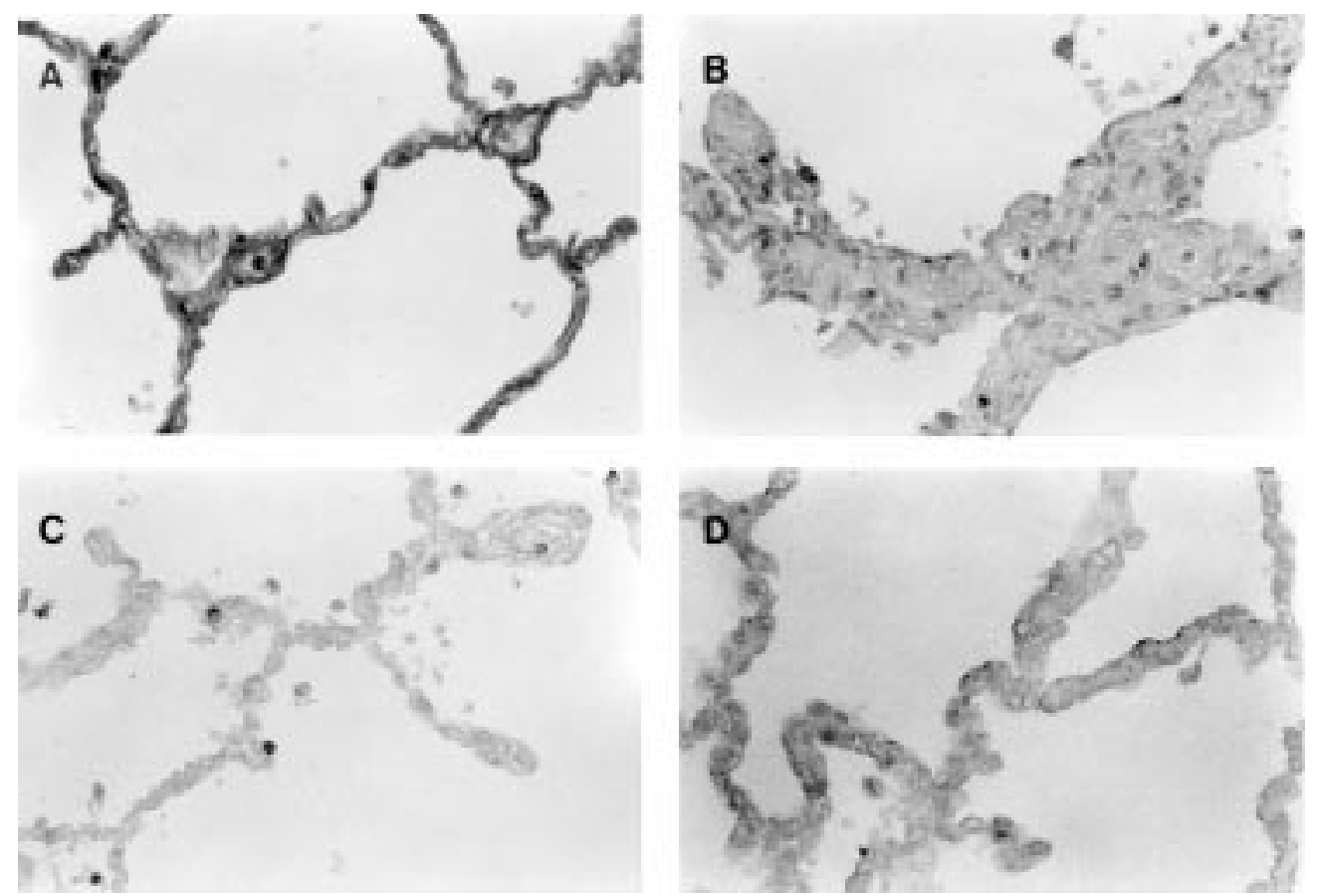

Figure 2 (A) Emphysematous lung showing prominent immunostaining (grade 4) with anti-lysozyme antibody. (B) Fibrotic lung showing focal immunostaining (grade 1) with anti-lysozyme antibody. (C) Normal lung showing focal immunostaining with anti-lysozyme antibody (grade 0), only associated with mononuclear inflammatory cells. (D) Normal lung control not treated with anti-lysozyme antibody showing complete lack of immunostaining. Streptavidin-biotin labelling counterstained with Mayer's haematoxylin. Original magnification $\times 800$.

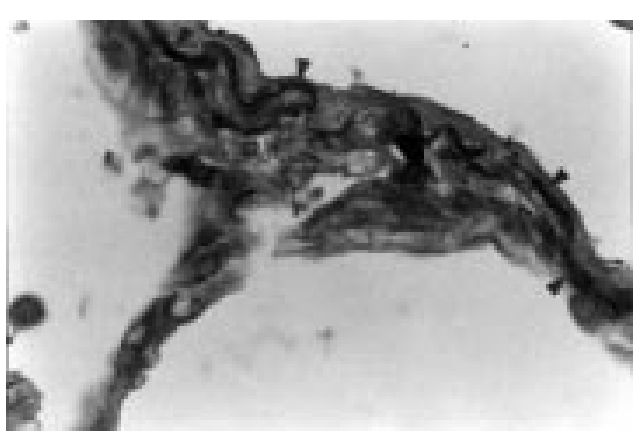

Figure 3 Disrupted elastic fibres (arrowheads) in emphysematous lung showing prominent immunostaining with anti-lysozyme antibody. Streptavidin-biotin labelling counterstained with Mayer's haematoxylin. Original magnification $\times 2000$.

against both human neutrophil lysozyme and egg white lysozyme.

\section{Discussion}

Determining the significance of any changes in lung lysozyme content is complicated by the fact that this protein has no recognised physiological function beyond its role in bacteriolysis. The increased levels of lysozyme which accompany inflammation may only reflect non-specific release from lysosomes. Furthermore, the association of lysozyme with inflamed tissues may be due to its strongly cationic nature, reflecting electrostatic binding to other tissue components rather than substrate-specific interactions.

Nevertheless, the presence of lysozyme in a wide variety of tissues suggests that it may have a physiological function. In cartilage, for example, lysozyme is present as a non-lysosomal matrix protein capable of interacting with surrounding polyanionic tissue components. Fur- thermore, it has been shown that injury to cartilage results in a parallel release of lysozyme and chondroitin sulphate, indicating a possible biochemical relationship between these two constituents. ${ }^{6}$ Since neither of these matrix components is specific to cartilage, similar interactions could occur in other tissues.

While the mechanism responsible for the observed affinity of lysozyme for elastic fibres is unclear, it is possible that lysozyme may bind to specific carbohydrate residues in elastic fibres. $\mathrm{N}$-acetyl-D-glucosamine, a component of bacterial cells susceptible to degradation by lysozyme, has also been found in glycoproteins associated with elastic fibres. ${ }^{7}$ Injury to elastic fibres, as occurs in pulmonary emphysema, ${ }^{8}$ may expose such residues, thereby facilitating lysozyme binding.

A study from this laboratory has previously shown that intratracheally administered hyaluronic acid preferentially binds to pulmonary elastic fibres and reduces alveolar injury experimentally induced by human neutrophil elastase. ${ }^{4}$ Hyaluronidase treatment of lung tissues may therefore increase lysozyme binding by degrading hyaluronic acid and exposing specific attachment sites on the elastic fibre. With regard to pulmonary emphysema, the presence of lysozyme on elastic fibres might disrupt the normal interactions between these fibres and other matrix components and possibly result in more rapid progression of the disease.

The presence of increased lysozyme in emphysematous tissues also lends further support to the concept that the disease is caused by the release of enzymes from neutrophils and monocytes. Since lysozyme is secreted by both neutrophils and monocytes, its presence on 
elastic fibres may serve as a "footprint" denoting the proximity of these cells to the fibres. Whether or not lysozyme facilitates the access of these cells to elastic fibres or alters the repair of the fibres remains to be determined.

1 Pruzanski W, Saito S, Orgryzlo $M$. The significance of lysozyme (muramidase) in rheumatoid arthritis. Arthritis Rheum 1970;13:389-99.

2 Mera SL, Lovell CR, Jones RR, et al. Elastic fibres in normal and sun-damaged skin: an immunohistochemical study. $\mathrm{Br}$ 7 Dermatol 1987;117:21-7.

3 Kahn HJ. "Aberrant elastic" in elastofibroma: an immunohistochemical and ultrastructural study. Ultrastruct Pathol 1995;19:45-50.
4 Cantor JO, Cerreta JM, Armand G, et al. Further investigation of the use of intratracheally administered hyaluronic acid to ameliorate elastase-induced emphysema. Exp Lung Res 1997;23:229-44

5 Baccarani-Contri M, Vincenzi D, Cicchetti F, et al. mmunocytochemical localization of proteoglycans within normal elastin fibers. Eur f Cell Biol 1990;53:305-12.

6 Greenwald RA, Cantor JO, Schwartz CE, et al. Effects of acute cartilaginous injury on serum and cartilage lysozyme levels. Arthritis Rheum 1975;18:139-44.

7 Amaya J. The effect of steroids on organ-cultured porcine trabecular meshwork: an ultrastructural, biochemical, and ectin histochemical study. Acta Soc Opthalmol fap 1995;99:995-1004.

8 Senior RM, Kuhn C III. The pathogenesis of emphysema. In: Fishman AP, ed. Pulmonary diseases and disorders. 2nd ed. New York: McGraw-Hill, 1982:1209-18. 